\title{
BLIND CHIP-RATE EQUALISATION FOR DS-CDMA DOWNLINK RECEIVER
}

\author{
S. Weiss, M. Hadef, M. Konrad \\ School of Electronics \& Computer Science \\ University of Southampton \\ Southampton, UK \\ $\{$ s.weiss,m. hadef $\} @$ ecs.soton.ac.uk
}

\author{
M. Rupp \\ Institute for Comms \& RF Engineering \\ Technical University of Vienna \\ Vienna, Austria \\ mrupp@nt .tuwien.ac .at
}

\begin{abstract}
A dispersive channel in the DS-CDMA downlink destroys the orthogonality of the synchronous users' spreading sequences. In this paper, we aim to re-establish this orthogonality blindly by means of a common chip-level equaliser. The adaptation algorithm is based on a constant modulus criterion forcing the various user symbols onto a constant modulus, for which a stochastic gradient descent algorithm is derived. This algorithm is structurally similar to a multiple error filtered-X LMS type approach, whereby the equaliser input $\mathrm{CM}$ update is replaced by a spreading code filtered version. Various simulations demonstrating the algorithm's convergence and noise performance are presented.
\end{abstract}

\section{INTRODUCTION}

In a DS-CDMA downlink scenario, transmission over a dispersive channel destroys the mutual orthogonality of the codes which are used to multiplex the various users in the system. As a result, the received and code-demultiplexed user signals are subject not only to inter-symbol interference (ISI) due to channel dispersion but also to multiple access interference (MAI) due to the loss of code orthogonality.

A popular approach to suppress MAI and ISI one a user is the minimum output power (MOE) algorithm blindly cancelling MAI and ISI terms but passing the desired user by code-constraints $[1,2]$, which is essentially Frost's linearly constrained minimum variance beamformer [3]. Recovering several users at the same time exploits more knowledge of the system and has been performed blindly using a constant modulus (CM) criterion $[4,5,6]$, whereby the derived algorithms either neglect spreading [5] or the dispersiveness of the channel $[4,6]$. Non-blind multiuser schemes in turn are based either on the knowledge of a pilot $[7,8]$ or training sequences [9].

In this paper, we derive, based on the definition of a signal model in Sec. 2, a suitable CM cost function for a chip-level equaliser in Sec. 3. Sec. 4 presents a stochastic gradient algorithm, which is structurally similar to the mul- tiple error filtered-X LMS algorithm in [10]. The addition mutual decorrelation between the various decoded user signals as required in [5] and [11] can be neglected due to the code-filtering. Simulations of the proposed algorithm are presented in Sec. 5, and conclusions drawn in Sec. 6.

\section{SIGNAL MODEL}

We consider the DS-CDMA downlink system in Fig. 1 with multiple symbol-synchronous users, which for simplicity are assumed to have the same rate. The system is fully loaded with $N$ user signals $u_{l}[n], l=0(1) N-1$, which are code multiplexed using Walsh sequences of length $N$ extracted from a Hadamard matrix $\mathbf{H}$. The resulting chip rate signal, running at $N$ times the symbol rate, is further scrambled by $c[m]$ prior to transmission over a channel with dispersive impulse response $g[\mathrm{~m}]$ and corruption by additive white Gaussian noise $v[m]$, which is assumed to be independent of the transmitted signal.

The dispersive channel $g[\mathrm{~m}]$ destroys the orthogonality of the Walsh codes, such that direct decoding of the received signal $r[m]$ with descrambling by $c^{*}[m]$ and code-matched filtering by $\mathbf{H}^{\mathrm{T}}$ will lead to MAI and ISI corruption of the decoded user signals $\hat{u}_{l}[n], l=0(1) N-1$. In order to re-establish orthogonality of the codes, a chip rate equaliser $w[m]$ can be utilised $[9,8]$. In the following, we are concemed with the blind updating of the equaliser coefficients

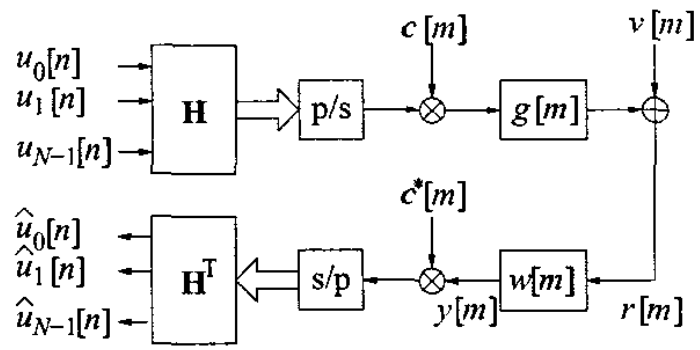

Fig. 1. DS-CDMA downlink signal model. 
$w[m]$.

\section{MULTIUSER EQUALISATION CRITERION}

We first derive the detected user signals $\hat{u}_{l}[m]$ as a function of the chip-rate equaliser $w[m]$. Based on this, we state a suitable cost functions based on which the equaliser can be adapted.

\subsection{Demultiplexed User Signals}

For the decoding, Walsh sequences are used as matched filters. The sequence for decoding the $l$ th user, contained in a vector $\mathbf{h}_{l}$, can be taken from an $N \times N$ Hadamard matrix,

$$
\mathbf{H}^{\mathrm{T}}=\left[\begin{array}{llll}
\mathbf{h}_{0} & \mathbf{h}_{1} & \cdots & \mathbf{h}_{N-1}
\end{array}\right]^{\mathrm{T}} .
$$

The $l$ th user is thus decoded as

$$
\begin{aligned}
& \hat{u}_{l}[n]=\mathbf{h}_{l}^{\mathrm{T}} \cdot\left[\begin{array}{cc}
c^{*}[n N] & \mathbf{0} \\
c^{*}[n N-1] & \\
\vdots & \ddots \\
0 & c^{*}[n N-N+1]
\end{array}\right] \cdot\left[\begin{array}{c}
y[n N] \\
y[n N-1] \\
\vdots \\
y[n N-N+1]
\end{array}\right] \\
& =\tilde{\mathbf{h}}_{l}^{\mathrm{T}}[n N] \cdot\left[\begin{array}{ccc}
\mathbf{w}^{\mathrm{H}} & & \mathbf{0} \\
& \mathbf{w}^{\mathrm{H}} & \\
& \ddots & \\
0 & & \mathbf{w}^{\mathrm{H}}
\end{array}\right] \cdot\left[\begin{array}{c}
r[n N] \\
r[n N-1] \\
\vdots \\
r[n N-L-N+2]
\end{array}\right]
\end{aligned}
$$

whereby the descrambling code $c^{*}[m]$ has been absorbed into a modified and now time-varying code vector $\tilde{\mathbf{h}}_{l}[n N]$, and $\mathrm{w} \in \mathbb{C}^{L}$ contains the equaliser's $L$ chip-spaced complex conjugate weights. Rearranging $\mathbf{w}$ and $\tilde{\mathbf{h}}_{l}[n N]$ yields

$$
\begin{aligned}
\hat{u}_{l}[n] & =\mathbf{w}^{\mathrm{H}} \cdot\left[\begin{array}{cc}
\tilde{\mathbf{h}}_{l}^{\mathrm{T}}[n N] & \mathbf{0} \\
& \tilde{\mathbf{h}}_{l}^{\mathrm{T}}[n N] \\
& \ddots \\
& \\
0 & \overline{\mathbf{h}}_{l}^{\mathrm{T}}[n N]
\end{array}\right]\left[\begin{array}{c}
r[n N] \\
r[n N-1] \\
\vdots \\
r[n N-L-N+2]
\end{array}\right] \\
= & \mathbf{w}^{\mathrm{H}} \mathbf{H}_{l}[n N] \mathbf{r}_{n N},
\end{aligned}
$$

with $\mathbf{H}_{l}[n N] \in \mathbb{Z}^{L \times(N+L-1)}$ being a convolutional matrix comprising the $l$ th user's modified code vector $\tilde{\mathbf{h}}^{\mathrm{T}}[n]$ and $\mathbf{r}_{n N} \in \mathbb{C}^{N+L-1}$.

\subsection{CMA Cost Function}

We assume that the user signals $u_{l}[m]$ consist of symbols with a constant modulus $\gamma$, such as BPSK, QPSK, or 8PAM. Therefore, we would like to blindly adapt the equaliser by forcing all decoded users $\hat{u}_{l}[\mathrm{~m}]$ onto a constant modulus. This can be formulated, similarly to $[5,12]$, by a suitable cost function $\xi_{\mathrm{CM}}$,

$$
\xi_{\mathrm{CM}}=\mathcal{E}\left\{\sum_{l=0}^{N-1}\left(\gamma^{2}-\left|\hat{u}_{l}[n]\right|^{2}\right)^{2}\right\},
$$

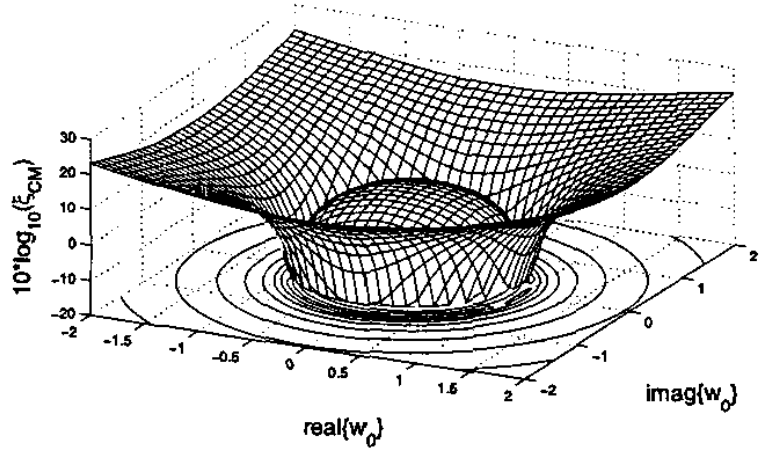

Fig. 2. Cost function $\xi_{\mathrm{CM}}$ in dependency of a single complex valued coefficient $w_{0}$.

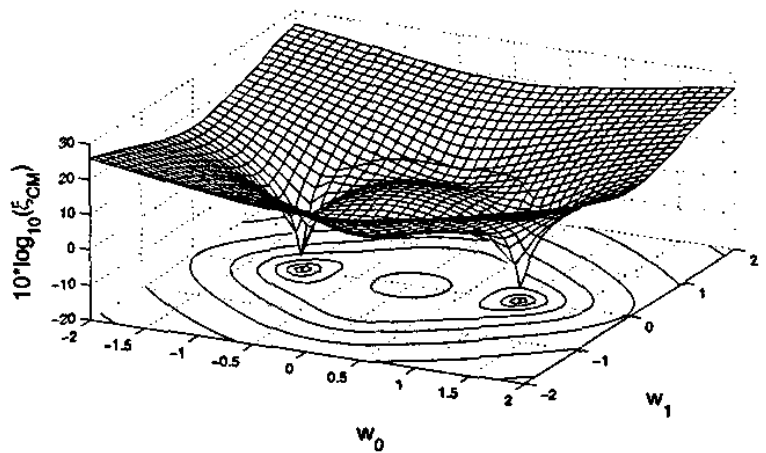

Fig. 3. Cost function $\xi_{\mathrm{CM}}$ in dependency of a two coefficient equaliser $\mathbf{w}=\left[\begin{array}{ll}w_{0} & w_{1}\end{array}\right]^{\mathrm{T}}$, which is constrained to be real.

which measures deviation of each of the $N$ users' decoded symbols from the desired modulus. The optimum equaliser coefficient vector $w$ is therefore given by

$$
\mathbf{w}_{\mathrm{opt}, \mathrm{CM}}=\arg \min _{\mathbf{w}} \xi_{\mathrm{CM}}
$$

There is no unique solution to (4), since minimising (3) is ambiguous with a manifold of solutions due to an indeterminism in phase rotation. However, any member of this manifold is a suitable solution for the equaliser $\mathbf{w}$, and can be used in combination with differential modulation schemes to recover $u_{l}[\mathrm{~m}]$.

Examples. Two examples for $\xi_{\mathrm{CM}}$ with $N=4$ users employing QPSK with $\gamma=1$ over a distortionless and delayless noise-free channel are given in Figs. 2 and 3. Fig. 2 shows $\xi_{\mathrm{CM}}$ in dependency of an equaliser $w$ with a single complex coefficient $w_{0}$. The cost function shows that there is as manifold of solutions satisfying $\left|w_{0}\right|=\gamma$.

Fig. 3 presents a case, where the equaliser contains two coefficients which are constrained to be real, and therefore possesses two symmetric solutions $\mathbf{w}_{\mathrm{opt}, \mathrm{CMA}}=\left[\begin{array}{ll} \pm 1 & 0\end{array}\right]^{\mathrm{T}}$ only. Note that a solution $\mathbf{w}=\left[\begin{array}{ll}0 & \pm 1\end{array}\right]^{\mathrm{T}}$ would not syn- 
chronise the codes correctly and therefore has a large cost function value associated. It is also apparent from Figs. 2 and 3 that $\xi_{\mathrm{CM}}$ has flat points, which we will discuss below.

\section{BLIND ADAPTATION}

A simple stochastic gradient descent update rule for $w[m]$ can be found by calculating the gradient of an instantaneous cost function, i.e. omitting the expectation operator in (3),

$$
\hat{\xi}_{\mathrm{CM}}=\sum_{l=0}^{N-1}\left(\gamma^{2}-\left|\hat{u}_{l}[n]\right|^{2}\right)^{2} .
$$

The resulting terms are then minimised w.r.t. $\mathbf{w}$ to obtain instantaneous estimates of the cost function gradient $\nabla \hat{\xi}\left(\mathbf{w}_{n}\right)$, leading to the stochastic gradient update

$$
\mathbf{w}_{n+1}=\mathbf{w}_{n}-\mu \nabla \hat{\xi}_{\mathrm{CM}}\left(\mathbf{w}_{n}\right)
$$

where $\mu$ is the step size. The introduction of gradient noise through inaccurate estimates of the true underlying statistics into the update routine can assist in avoiding the adaptation to remain in flat points of the cost function.

To determine $\nabla \hat{\xi}_{\mathrm{CM}}$, we apply complex vector calculus [13] to (5), yielding

$$
\begin{aligned}
\frac{\partial \hat{\xi}_{\mathrm{CM}}}{\partial \mathbf{w}^{*}} & =-2 \sum_{l=0}^{N-1}\left[\left(\gamma^{2}-\left|\hat{u}_{l}[n]\right|^{2}\right) \frac{\partial}{\partial \mathbf{w}^{*}} \hat{u}_{l}[n] \hat{u}_{l}^{\mathrm{H}}[n]\right] \\
& =-2 \sum_{l=0}^{N-1}\left[\left(\gamma^{2}-\left|\hat{u}_{l}[n]\right|^{2}\right) \mathbf{H}_{l}[n N] \mathbf{r}_{n N} \cdot\right. \\
\left.\cdot \mathbf{r}_{n N}^{\mathrm{H}} \mathbf{H}_{l}^{\mathrm{H}}[n N] \mathbf{w}\right] & \\
= & -2 \sum_{l=0}^{N-1}\left(\gamma^{2}-\left|\hat{u}_{l}[n]\right|^{2}\right) \mathbf{H}_{l}[n N] \mathbf{r}_{n N} \hat{u}_{l}^{*}[n]
\end{aligned}
$$

This algorithm differs from the standard CM algorithm [14] or its extension in [5] in the inclusion of a code filtered term $\mathbf{H}_{l}[n N] \mathbf{r}_{n N}$ rather than just the equaliser input $r[n]$. This is structurally similar to a multiple-error filtered-X LMS algorithm [10], where the transfer functions appearing in the
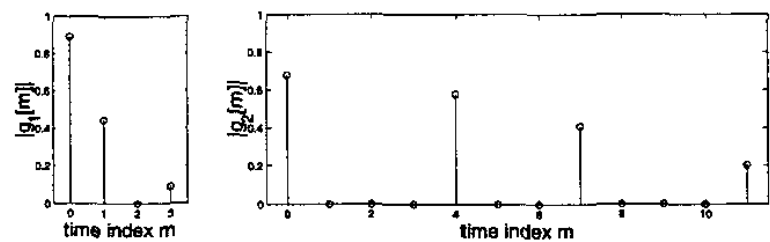

Fig. 4. Moduli of complex valued channel impulse responses $g_{1}[m]$ (left) and $g_{2}[m]$ (right). paths between the adaptive filter output and the error formations have to be accounted for by modifying the LMS' updating scheme. Hence, we refer to the proposed scheme in (7) as filtered-R multiple error CM algorithm (FIRMERCMA).

\section{SIMULATION RESULTS}

For the simulations below, we apply the FIRMER-CMA to two different channel impulse responses, a short $g_{1}[\mathrm{~m}]$ and a more dispersive $g_{2}[m]$, as characterised in Fig. 4. We first demonstrate the convergence behaviour in Sec. 5.1, and later the bit error performance in Sec. 5.2.

\subsection{Convergence}

In order to demonstrate the convergence behaviour of the proposed algorithm, we transmit $N=16$ QPSK user signals over $g_{1}[\mathrm{~m}]$ in the absence of channel noise, and utilise the FIRMER-CMA to update an equaliser with 10 coeffcients. The adaptation is initialised with the second coefficient in the weight vector set to unity, while the optimal response will need to place the maximum coefficient in the first vector element. With $\mu=0.05$, the evolution of the filter coefficients' real part is shown in Fig. 5(top), whereby the instantaneous cost function $\hat{\xi}_{\mathrm{CM}}$ of $(5)$ is given in Fig. 5(bottom). The system converges to a solution such that the convolution of $g_{1}[\mathrm{~m}]$ and the adapted equaliser is a complex rotation of a zero delay. This rotation is evident when considering the evolution of the constellation pattern of the demodulated 0th user $u_{0}[n]$ as presented in Fig. 6 . Although adaptation is switched on at $n=0$, the system exhibits a slow initial convergence which may be due to the flat points in $\xi_{\mathrm{CM}}$, but is generally typical for CMA algorithms [14].

We now assume a carrier offset $\Delta \Omega$, such that a modified baseband channel is given by

$$
\tilde{g}_{1}[m]=g_{1}[m] e^{j \Delta \Omega n}
$$

whereby here $\Delta \Omega=0.510^{-5} \pi$. Converging the FIRMERCMA on this system yields symbols streams as exemplified for user $l=0$ in Fig. 7. As the dynamic rotation applied to the baseband model does not affect the CM criterion, and the performance of FIRMER-CMA appears unaffected by a carrier offset.

The problem of rotating user signals is not further consider here, but could be addressed, for example, by carrier offset estimation in a second receiver stage analogously to $[15]$.

\subsection{Robustness}

For $N=4$ users, we have adapted the FIRMER-CMA under various SNR conditions for the channel impulse re- 

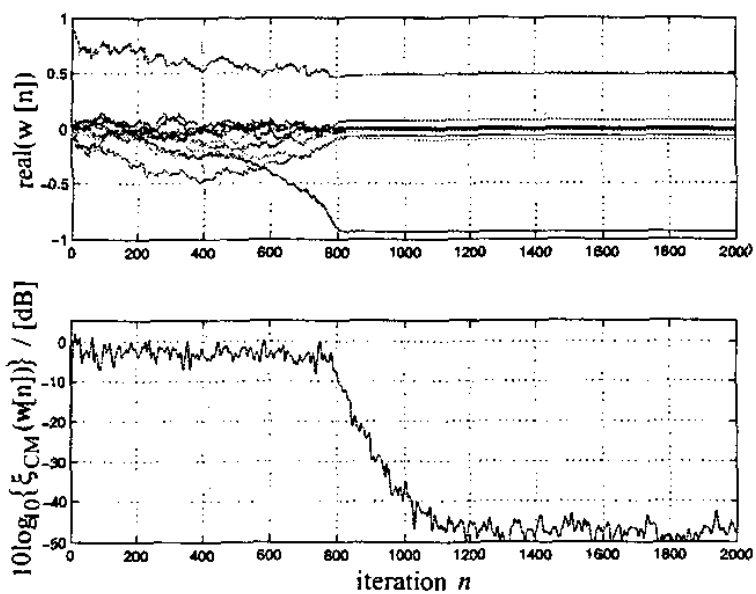

Fig. 5. (top) weight trajectories and (bottom) learning curve.

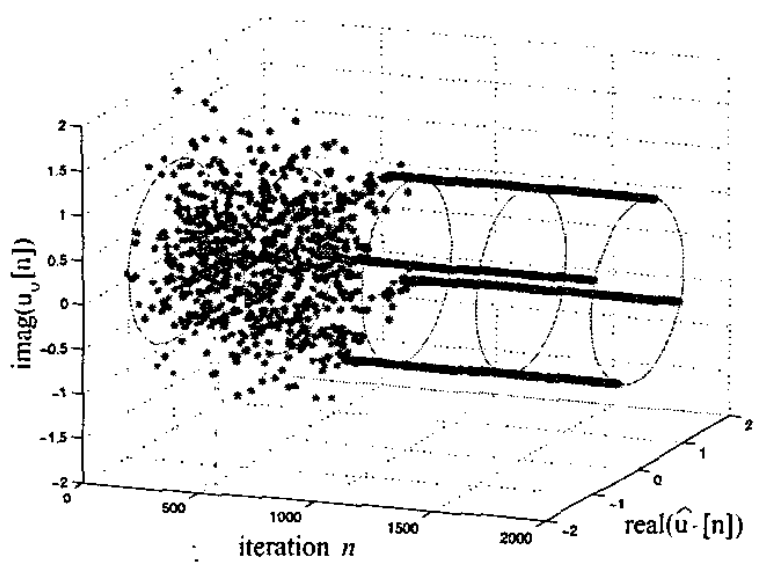

Fig. 6. Evolution of the received constellation pattern of user $l=0$ over a stationary channel $g_{1}[m]$.

sponses $g_{1}[m]$ and $g_{2}[m]$ with $L=20$ and $L=64$ coefficients, respectively. With the middle tap set to unity and an appropriately adjusted $\mu$, the algorithm has always been given $10^{3}$ symbol periods to converge prior to correction of the phase rotation and bit error rate (BER) measurement. The BER results are given in Fig. 8 in comparison to the optimal QPSK performance in AWGN and the analytical minimum MSE (MMSE) solution. Note that the FIRMERCMA closely approaches the MMSE performance.

In order to explore the steady-state performance of the system, channel $g_{2}[\mathrm{~m}]$ in Fig. 4(right) has been used as an average magnitude profile. Each of the 4 multipath coefficients is now drawn from a Rayleigh distribution. We use this system to explore the influence of the step size parameter $\mu$ on the convergence. Allowing the FIRMER-CMA

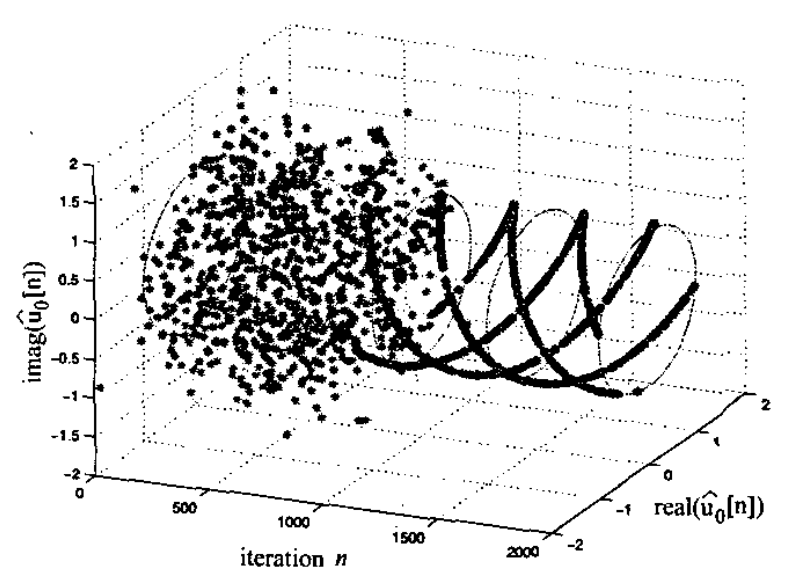

Fig. 7. Evolution of the received constellation pattern of user $l=0$ over a channel $\tilde{g}_{1}[m]$ with carrier offset.

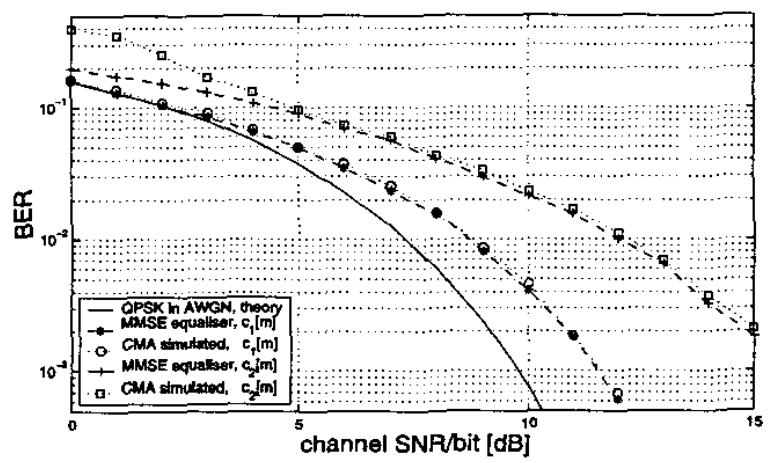

Fig. 8. BER performance of proposed FIRMER-CMA over two channels in dependency of the channel SNR compared to an analytical MMSE channel equaliser and the BER in an AWGN channel.

sufficient time to reach its steady state for each of 200 independent, fixed channel realisations, the various BER curves for rotation-corrected systems are compared to their analytic MMSE equaliser performances. The later are chosen to realise a delay of $L / 2=32$ chips for every channel, while the FIRMER-CMA is initialised with the centre coefficient set to unity. The results for step sizes of $\mu=0.001, \mu=0.0003$, and $\mu=0.0001$ are given in Fig. 9, and compared to the theoretical performance of QPSK over a non-dispersive AWGN channel, as well the the MMSE BER curve. It can be seen that for small values of $\mu$, the steady-state performance of FIRMER-CMA approaches the MMSE performance closely, while for larger $\mu$, the performance in noise is generally seriously degraded, particularly for lower BER performances in higher SNR regions. 


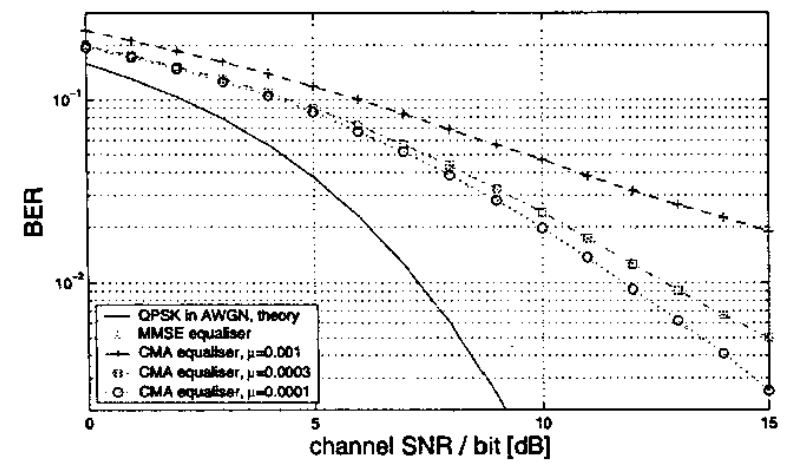

Fig. 9. BER performance of proposed FIRMER-CMA over two channels in dependency of the channel SNR compared to an analytical MMSE channel equaliser and the BER in an AWGN channel.

\section{CONCLUSIONS}

A blind equalisation approach for a DS-CDMA downlink scenario has been presented, which aims to enforce CM conditions on the various user signals. A stochastic gradient algorithm has been derived, which differs from previous $\mathrm{CM}$ algorithms by a code-prefiltering of its input. Compared to other multiuser $\mathrm{CM}$ based solutions in the literature $[5,11]$, the spreading codes enforce the orthogonality of the decoded user, which renders decorrelation of the various output signals obsolete. The proposed algorithm has been extensively tested and proven very stable. Representative simulations have been presented, highlighting the convergence behaviour as well as the algorithm's noise performance, which is sensitive to the selection of the step size parameter but can reach very close to the performance of the MMSE equaliser.

\section{REFERENCES}

[1] M. Honig, U. Madhow, and S. Verdu, "Blind Adaptive Multiuser Detection," IEEE Trans. Inf. Theory, 41(4):944-960, 1995.

[2] R. Schober, W. H. Gerstacker, and L. H. J. Lampe, "Comparison of MOE and blind LMS," IEEE Comms. Lett., 7(5):204-206, 2003.

[3] O. L. Frost, III, "An Algorithm for Linearly Constrained Adaptive Array Processing," Proc. IEEE, 60(8):926-935, 1972.

[4] K. Fukawa and H. Suzuki, "Orthogonalizing Matched Filter (OMF) Detection for DS-CDMA Mobile Radio Systems," in IEEE GlobeCom, 1994, 1: 385-389.
[5] C. Papadias and A. Paulraj, "A Constant Modulus Algorithm for Multiuser Signal Separation in Presence of Delay Spread Using Antenna Arrays," IEEE Sig. Proc. Lett., 4(6):178-181, 1997.

[6] Y. Huang and P. M. Djurić, "Multiuser Detection of Synchronous Code-Division Multiple-Access Signals by Perfect Sampling," IEEE Trans. Sig. Proc., 50(7): 1724-1734, 2002.

[7] F. Petre, M. Moonen, M. Engels, B. Gyselinckx, and H.D. Man, "Pilot-Aided Adaptive Chip Rate Equalizer Receiver for Interference Suppression in DS-CDMA Forward Link," in IEEE VTC, Sept. 2000, 1:303-308.

[8] A. R. Margetts and P. Schniter, "Adaptive ChipRate Equalization of Downlink Multirate Wideband CDMA," IEEE Trans. Sig. Proc., to appear, 2004.

[9] I. Stirling, D. Garcia-Alis, S. Weiss, G. Rice, and R.W. Stewart, "A Joint Adaptive MMSE Downlink Receiver," in Asilomar Conf. Sig. Sys. Comp., Nov. 2000. 2:1436-1440.

[10] S.J. Elliott, I.M. Stothers, and P.A. Nelson, "A Multiple Error LMS Algorithm and its Application to the Active Control of Sound and Vibration," IEEE Trans. Acoustics, Speech Sig. Proc., 35(10):1423$1434,1987$.

[11] S. Lambotharan, J. A. Chambers, and A. G. Constantinides, "Adaptive Blind Retrieval Techniques for Multi-User DS-CDMA Signals," IEE Electronics Letters, 35(9):693-695, 1999.

[12] Z. Xu and P. Liu, "Code-Constrained Blind Detection of CDMA Signals in Multipath Channels," IEEE Sig. Proc. Lett., 9(12):389-392, 2002.

[13] T. K. Moon and W. C. Stirling, Mathematical Methods and Algorithms, Prentice Hall, 1999.

[14] C. R. Johnson, P. Schniter, T. J. Endres, J. D. Behm, D. R. Brown, and R. A. Casas, "Blind Equalization Using the Constant Modulus Criterion: A Review," Proc. IEEE, 86(10): 1927-1950, 1998.

[15] M. Rupp, "On the Separation of Channel and Frequency Offset Estimation," in Asilomar Conf. Sig. Syst. Comp., Monterey, CA, October 1998, 2:1186-1190. 